\title{
Crystalline Morphology and Melting Behavior of 100\% Syndiotactic Polystyrene and 70/30 Blend of Syndiotactic Polystyrene and Poly(2, 6-dimethyl-1, 4-phenylenoxide)
}

\author{
Soon Man HonG, ${ }^{*}$ Soo Hyun CHOI, ${ }^{* *}$ Chang Hyung LeE, ${ }^{\dagger}$ Seung Sang HwANG, ${ }^{* *}$ \\ Kwang Ung KIM, ${ }^{* *}$ and Iwhan $\mathrm{CHO}^{*}$ \\ Department of Medical Devices \& Radiation Health, Korea Food \& Drug Administration \\ 5, Nokbeon-dong, Eunpyung-ku, Seoul 122-701, Korea \\ *Polymer Processing Laboratory, Polymer Hybrid Research Center, Korea Institute of Science and Technology (KIST) \\ 39-1, Hwawolk-dong, Songbuk-ku, Seoul 136-791, Korea \\ ** Department of Advanced Materials Engineering (Polymerics Program), Korea Advanced Institute of Science and Technology (KAIST) \\ P. O. Box 201, Cheongryangri-dong, Dongdaemoon-ku, Seoul 130-650, Korea
}

(Received February 24, 1999)

\begin{abstract}
The thermal behavior of isothermally crystallized syndiotactic polystyrene (SPS) and 70/30 SPS/poly (2,6-dimethy 1-1,4-diphenylene oxide) (PPO) blend was investigated by differential scanning calorimetry. Pure SPS, which was crystallized at $T_{\mathrm{c}}=220$ and $240^{\circ} \mathrm{C}$ had single melting endotherm and multiple endotherms, respectively, whereas the 70/30 SPS/PPO blends showed the opposite behavior with respect to $T_{\mathrm{c}}$. Investigation by polarizing optical microscopy and small angle X-ray scattering revealed that the rejected impurity (PPO in blend and noncrystallizable chains with low molecular weight in pure SPS) during SPS crystallization resides between primary lamellae of SPS and thus the recrystallization strongly suppressed for the pure SPS crystallized at $T_{\mathrm{c}}=220^{\circ} \mathrm{C}$ and the $70 / 30 \mathrm{SPS} / \mathrm{PPO}$ blend crystallized at $T_{\mathrm{c}}=240^{\circ} \mathrm{C}$. This may induce single melting endotherm. In the pure SPS crystallized at $T_{\mathrm{c}}=240^{\circ} \mathrm{C}$ and the $70 / 30 \mathrm{SPS} / \mathrm{PPO}$ blend crystallized at $T_{\mathrm{r}}=220^{\circ} \mathrm{C}$, the rejected impurity resided between SPS crystalline fibrils, which consist of lamellae. This may allow recrystallization between the primary lamellae which would exhibit sharp multiple melting endotherms.

KEY WORDS Syndiotactic Polystyrene / Crystalline Morphology / Multiple Endotherm / Small-Angle X-Ray Scattering /
\end{abstract}

Semicrystalline syndiotactic polystyrene (SPS) has a high melting temperature, $270^{\circ} \mathrm{C}$ and good chemical resistance due to its tacticity. These properties have been recently studied in detail, with emphasis on the crystalline structures of these polymers. It is now possible to get a SPS using the metallocene/methylaluminoxane catalyst system. ${ }^{1}$ Since SPS with a very high degree of stereo-specificity $(>96 \%)$ has been synthesized by stereospecific polymerization, there has been considerable interest in the characteristics of this new material. ${ }^{2-4}$ Improved mechanical properties are expected for SPS, especially at high temperature, due to its crystalline structure. We expect a fast crystallization rate for SPS compared with that of isotactic polystyrene and that its high tensile modulus will make it a potential engineering plastic.

Poly(2,6-dimethyl-1,4-diphenylene oxide) (PPO) is an amorphous material with high glass transition temperature $\left(T_{\mathrm{g}}=205^{\circ} \mathrm{C}\right)$. It has excellent impact strength even at very low temperatures. PPO shows a good dimensional stability due to low mold shrinkage. However, it is expensive and requires high processing temperature. Taking these into account, it is expected that the two polymers complement each other in a blend, overcoming the drawbacks, while maintaining good properties.

The blends of SPS/PPO are found to be molecularly miscible at all compositions. ${ }^{5}$ In miscible semicrystalline polymer/amorphous polymer blend, the amorphous component influences the crystallization of the semicrystalline polymer ; in SPS/PPO blend, the noncrystalline PPO

\footnotetext{
${ }^{\dagger}$ To whom all correspondence should be addressed.
}

could have a significant influence on the crystalline structure on the scale of several tens $\mathrm{nm}$ to several tens $\mu \mathrm{m}$. Therefore, we need to know the effects of the PPO and melting behavior on SPS crystalline structure, which should be related to physical properties of the materials.

\section{EXPERIMENTAL}

\section{Blends Procedures}

A special grade of SPS powder (Idemitsu Kosan Co. Ltd ; $M_{w}=4 \times 10^{5}$ ) was used. PPO powder was supplied by General Electric Co. (G 646-111; $\left.M_{w}=5.5 \times 10^{5}\right)$. Both powders were dried in a vacuum oven for $24 \mathrm{~h}$ at $50^{\circ} \mathrm{C}$. SPS/PPO blends were prepared by solution casting from $o$-dichlorobezene at about $170^{\circ} \mathrm{C}$. The blended samples were molded into $1.5 \mathrm{~mm}$-thick films between two steel plates with Teflon space bar on a heating stage; the stage was held at $300^{\circ} \mathrm{C}$ for $3 \mathrm{~min}$, to erase the previous thermal history, and the samples were quenched into ice water.

\section{Polarizing Optical Microscopy and Differential Scanning Calorimetry (DSC)}

Spherulitic texture was observed with a polarizing optical microscopy (Nikon Optiphoto-2) equipped with a hot stage. Polarized optical microscopic observations on the morphology of SPS isothermally crystallized with or without PPO were carried out at $220^{\circ} \mathrm{C}$ and $240^{\circ} \mathrm{C}$. Concentration effect of the PPO on the morphology of SPS was also examined at above crystallization temperature condition $\left(T_{\mathrm{c}}\right)$. 
The thermal behavior of the pure SPS and 70/30 SPS/ PPO blend, after isothermal crystallization was examined by DSC. A Perkin-Elmer DSC-7 was used to obtain the DSC thermograms at a heating rate of $10^{\circ} \mathrm{C} \mathrm{min}-1$ and was calibrated with indium. The scans were run with samples ranging from 10 to $15 \mathrm{mg}$ from $30^{\circ} \mathrm{C}$ to 300 ${ }^{\circ} \mathrm{C}$ under a nitrogen purge to prevent oxidative degradation. $T_{\mathrm{g}}$ was obtained second running of $10^{\circ} \mathrm{C} \mathrm{min} \mathrm{m}^{-1}$ heating rate after melt at $300^{\circ} \mathrm{C}$ for $5 \mathrm{~min}$ and quenching in ice-water.

\section{Small-Angle X-Ray (SAXS) Measurements}

The X-ray beam was from synchrotron radiation; beam line 3C2 at Pohang Accelerator Laboratory, Pohang, Korea. The storage ring was operated at an energy level of $2 \mathrm{GeV}$. The SAXS employs a point-focusing optics with a Si double crystal monochromator followed by a bent cylindrical mirror. The incident beam intensity of $0.149 \mathrm{~nm}$ wavelength was monitored by an ionization chamber for the correction of minor decrease of the primary beam intensity during the measurement.

Scattering intensity, $I$, was corrected for background scattering. Scattering intensity by thermal fluctuations was subtracted from the SAXS profile $I(q)$ by evaluating the slope of a $I(q) q^{4}$ versus $q^{4}$ plots $^{6}$ at wide scattering vectors $q$, where $q$ is $(4 \pi / \lambda) \sin \theta, \lambda$ and $2 \theta$, the wavelength and scattering angle, respectively. The data were corrected by the Lorentz equation $\left(I^{2} v s . q\right)$, with the intensity extrapolated to $q=0$ and $\backsim$. The correction for smearing effect by the finite cross section of the incident beam was not necessary for the optics of SAXS with point focusing.

\section{RESULTS AND DISCUSSION}

\section{SPS/PPO Miscibility in the Amorphous State}

In Figure 1, the $T_{\mathrm{g}}$ of the SPS/PPO blends measured with DSC is plotted as a function of blend composition. $T_{\mathrm{g}}$ temperatures were measured on samples heated from $30^{\circ} \mathrm{C}$ to $300^{\circ} \mathrm{C}$ at $10^{\circ} \mathrm{C} \mathrm{min}{ }^{-1}$. The samples were then rapidly removed from the DSC cell and quenched in water bath to make a totally amorphous. The dotted line represents the Fox equation

$$
\frac{1}{T_{\mathrm{g}}}=\frac{w_{1}}{T_{\mathrm{g} 1}}+\frac{w_{2}}{T_{\mathrm{g} 2}}
$$

where $w_{1}$ and $w_{2}$ represent the weight fractions of the blend constituents and $T_{\mathrm{g} 1}$ and $T_{\mathrm{g} 2}$ their respective glass transition temperatures. The single $T_{\mathrm{g}}$ observed for the sample indicated that SPS and PPO are miscible in the amorphous state over the entire composition range and that the blend $T_{\mathrm{g}}$ obeys the FOX equation within experimental accuracy.

\section{Melting Behavior and Spherulite Growth Rate}

DSC heating traces of the pure SPS and 70/30 SPS/ PPO blend isothermally crystallized at $T_{\mathrm{c}}=220^{\circ} \mathrm{C}$ and $240^{\circ} \mathrm{C}$ for $20 \mathrm{~min}$ are shown in Figure 2. The blended sample crystallized at $T_{\mathrm{c}}=220^{\circ} \mathrm{C}$ has multiple melting endotherms, whereas, in case of $240^{\circ} \mathrm{C}$, pure SPS shows multiple melting endotherms. The multiple melting endotherms have been reported for annealed samples of polyamides, ${ }^{7}$ poly(ether ether ketone) ${ }^{8}$ poly(ethylene

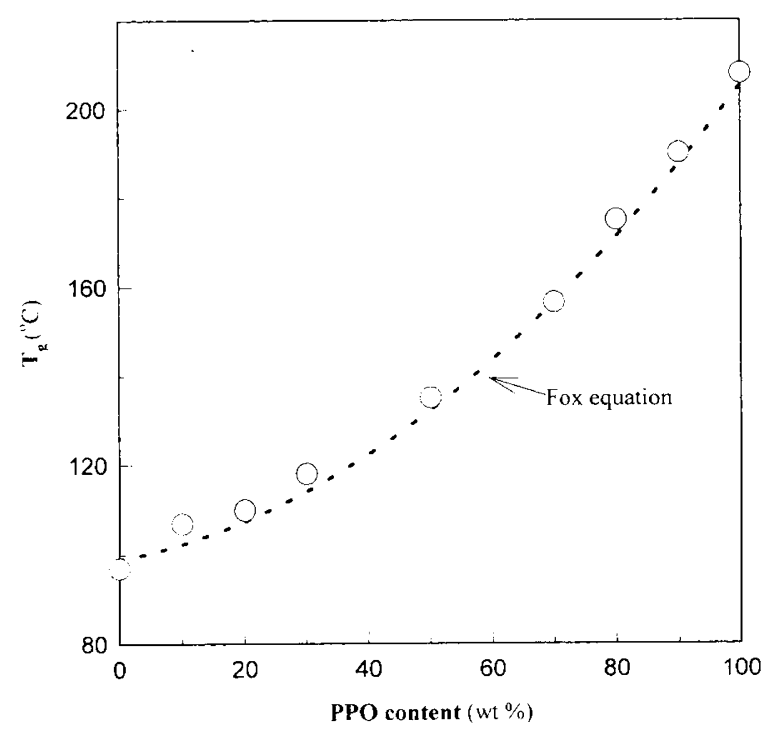

Figure 1. $T_{\mathrm{g}}$ of SPS/PPO blends versus composition.

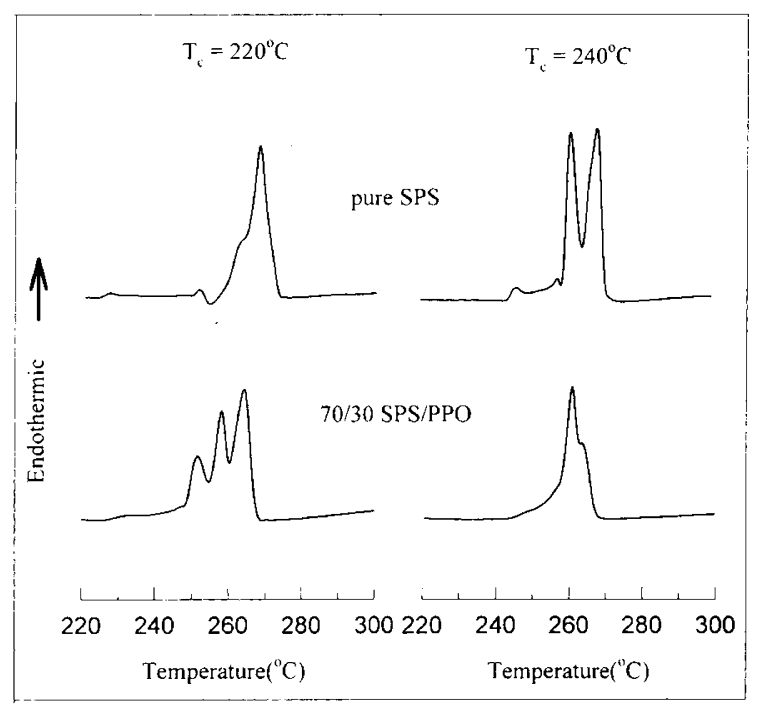

Figure 2. DSC melting thermograms of pure SPS and $70 / 30$ SPS/PPO blend $T_{\mathrm{c}}=220^{\circ} \mathrm{C}$ and $240^{\circ} \mathrm{C}$. Scan rate is $16^{\circ} \mathrm{C} \mathrm{min}-1$.

terephthalate) (PET), ${ }^{9}$ and low density polyethylene. ${ }^{10}$ The multiple melting behavior indicates partial melting $^{10}$ and recrystallization of the lower melting lamellae ${ }^{11}$; Higher melting lamellae are formed prior to the lower melting crystallites that lie between the primary lamellae.

At low crystallization temperature $\left(T_{\mathrm{c}}=220^{\circ} \mathrm{C}\right)$, pure SPS exhibits a sharp endotherm and a small shoulder. We designate this shape of the endotherm as single endotherm. At high crystallization temperature $\left(T_{\mathrm{c}}=240\right.$ ${ }^{\circ} \mathrm{C}$ ), it shows sharp multiple endotherms. Similar behavior was reported for isothermally annealed PET. ${ }^{9}$ To understand the melting endotherm, we investigated the time variation of the radius of SPS spherulites during isothermal crystallization with a polarizing optical microscopy.

The spherulite growth rate $G$ was obtained from the time variation of the spherulite radius and the results were shown in Figure 3. The melting behavior of the samples crystallized at different temperatures can be ex- 


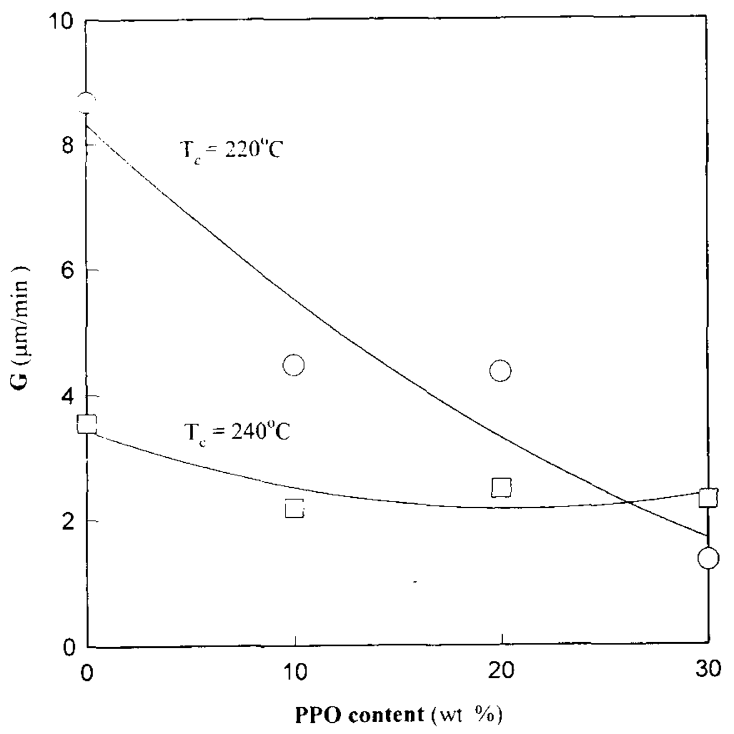

Figure 3. $G$ of SPS/PPO blends versus composition.

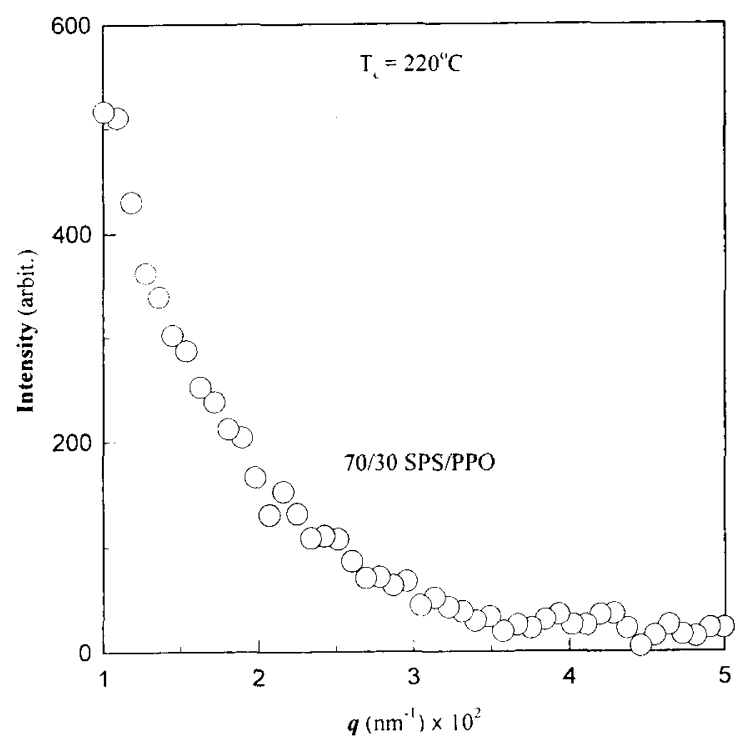

Figure 4. Typical SAXS profile for 70/30 SPS/PPO blend Crystallization time is $60 \mathrm{~min}$ at $T_{\mathrm{c}}=220^{\circ} \mathrm{C}$.

plained by considering the spherulite growth rate and participation of the impurities in the crystallization. The impurities defined here are noncrystallizable chains with low molecular weight in pure SPS and PPO chains in blends. At $T_{\mathrm{c}}=220^{\circ} \mathrm{C}$, the crystallization rate $G$ is high and the rejected impurity may be segregated between interlamellae. The higher content of the rejected impurity between lamellae prevents crystallization between the SPS primary lamellae, so that the endotherm, i.e., the fraction of the recrystallized lamellae, at low temperature would be very small. In general, the recrystallized lamellae are thin owing to space restriction and consequently they melt at lower temperature than primary lamellae. ${ }^{8}$

At $T_{\mathrm{c}}=240^{\circ} \mathrm{C}, G$ is slow and thus the rejected impurity may reside between fibrils consisting of lamellar bundle. The rejected impurity may be the noncrystallizable chains with low molecular weight in pure SPS. When an amount of impurity between interlamellae is small, it

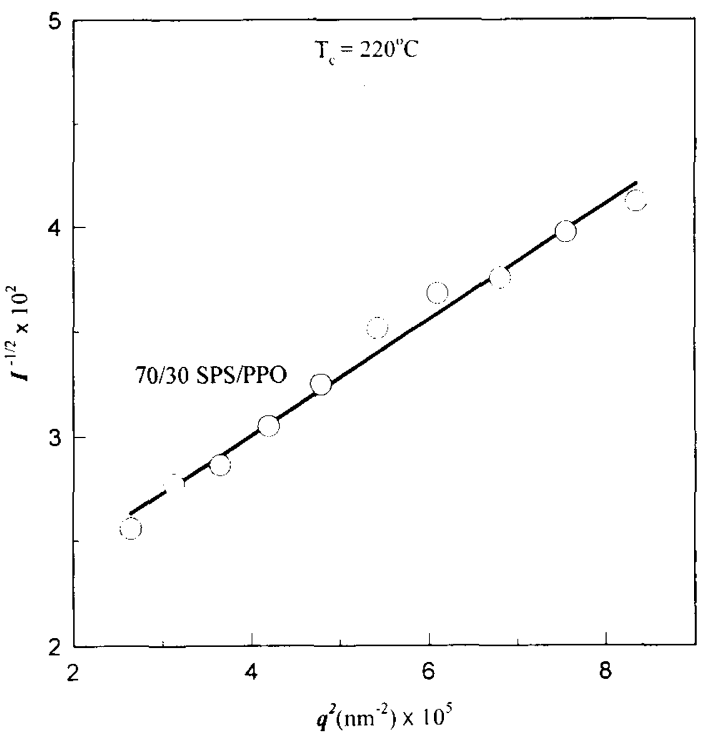

Figure 5. Typical Debye-Bueche plot for 70/30 SPS/PPO blend Crystallization time is $60 \mathrm{~min}$ at $T_{\mathrm{c}}=220^{\circ} \mathrm{C}$.

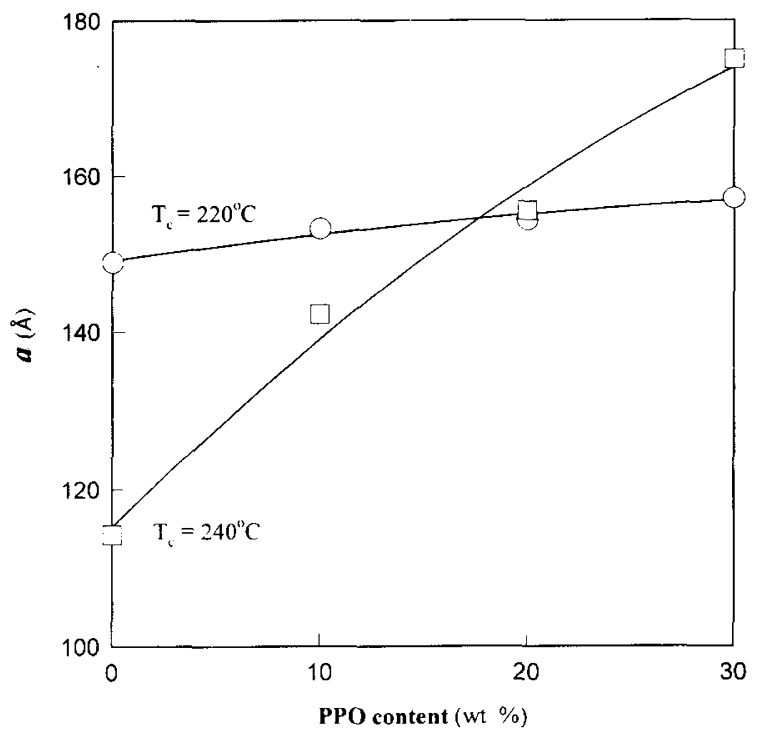

Figure 6. $a$ of SPS/PPO blends versus composition.

may be easy to recrystallized between interlamellae. The high fraction of the recrystallized lamellae can induce the sharp multiple melting endotherms.

By blending PPO, the tendency of the melting endotherms at low and high temperatures is reversible, compared with that in pure SPS (refer to Figure 2). This suggests that, at $T_{\mathrm{c}}=220^{\circ} \mathrm{C}$, the relative rates $(D / G)$ of the diffusion rate of non-crystalline component $(D)$ and the $G$ is comparable with the inter-fibril distance due to high $G$ and the impurity (PPO) resides at the inter-fibril region. This increases recrystallization between interlamellae and then sharp melting endotherms at low temperature is exhibited. At $T_{\mathrm{c}}=240^{\circ} \mathrm{C}$, the rejected impurity may reside between crystalline lamellae, since $D /$ $G$ is smaller because of high $G$. This prevents recrystallization between the primary lamellae and then sharp multiple melting endotherms appear. It is presently unclear why $D / G$ is so sensitive to the blend. 


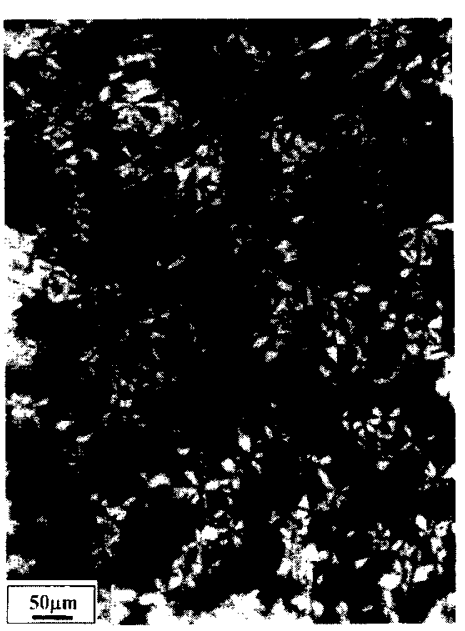

(a)

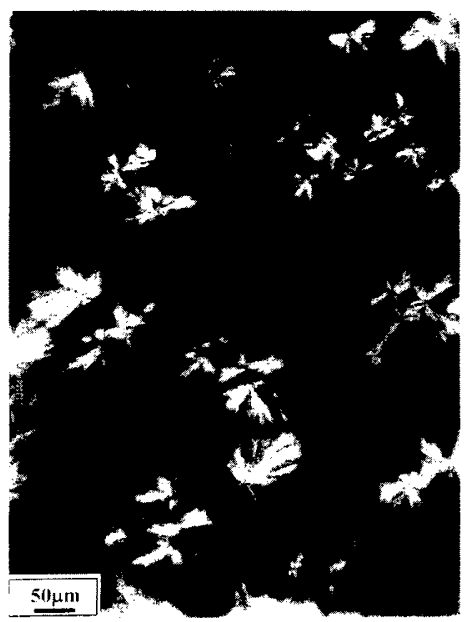

(b)

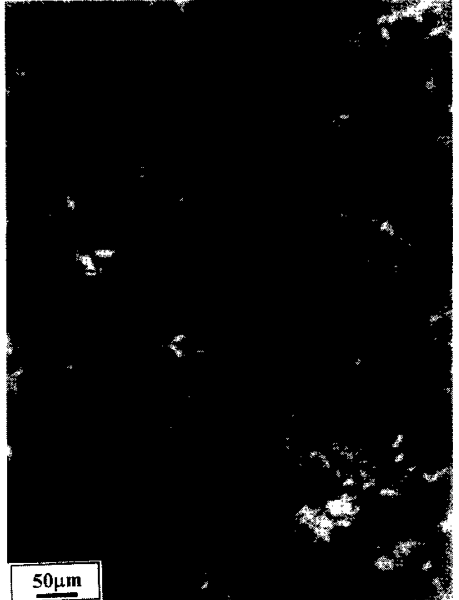

(a')

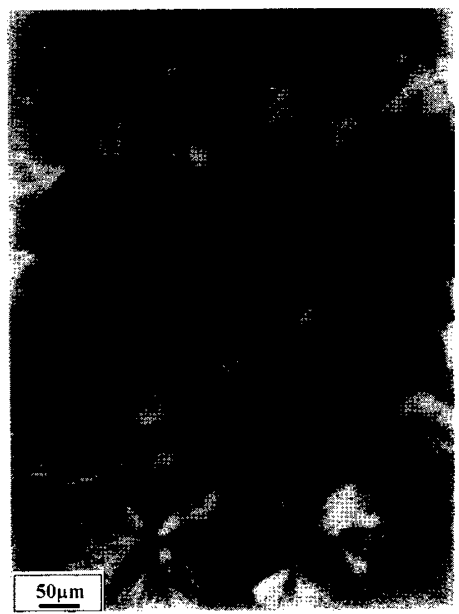

(b')

Figure 7. Polarized optical micrographs (a) for pure SPS crystallized at $T_{\mathrm{c}}=220^{\circ} \mathrm{C}$, (a') for pure SPS crystallized at $T_{\mathrm{c}}=240^{\circ} \mathrm{C}$. (b) for $70 / 30$ SPS/PPO blend crystallized at $T_{\mathrm{c}}=220^{\circ} \mathrm{C}$ and ( $\left.\mathrm{b}^{\prime}\right) 70 / 30$ SPS/PPO blend crystallized at $T_{\mathrm{c}}=240^{\circ} \mathrm{C}$.

Structure on the Crystalline Lamellae Level

Typical SAXS curve of 70/30 SPS/PPO blend isothermally crystallized at $T_{\mathrm{c}}=220^{\circ} \mathrm{C}$ is depicted in Figure 4 . For all samples tested, the scattering intensity decreased monotonously with $q$. The monotonously decreasing profile without scattering peak suggests irregular interlamellar arrangement. To analyze the scattering profile, the Debye-Bueche model, ${ }^{12}$ based on a simple exponential correlation function shown below, is normally applied to a random two phase system ; one is a SPS crystalline lamellar phase, and the other is an amorphous phase.

$$
\gamma(r)=\exp (-r / a)
$$

By Fourier transforming the exponential correlation function, the intensity profile is obtained and yields a Lorentzian function with a parameter $a$, defined as the correlation length, which is a measure of the size of heterogeneity.

$$
I^{-1 / 2}(q)=A+B q^{2}
$$

Where $A$ and $B$ are constants. Figure 5 shows a typical
Debye-Bueche plot. As expected from eq 2, the plot yields a straight line, indicating an irregular interlamellar arrangement. According to the above equation, the (slope/intercept) $)^{1 / 2}$, i.e., $(B / A)^{1 / 2}$ yields the correlation length $a$. The composition dependence of $a$ is plotted in Figure 6. On the basis of the magnitude of $a$ one can identify correlation length as correlation distance between SPS crystalline lamellae and amorphous phase. For pure SPS, $a$ is smaller at $T_{\mathrm{c}}=240^{\circ} \mathrm{C}$ than that at $T_{\mathrm{c}}$ $=220^{\circ} \mathrm{C}$. At sample crystallized at $T_{\mathrm{c}}=240^{\circ} \mathrm{C}$, the smaller a may indicate recrystallization between the primary lamellae. When a smaller amount of noncrystallizable chains (i.e., low molecular weight chains in pure SPS) is entrapped between SPS crystalline lamellae, it may be easy to recrystallize between primary lamellae and thus its recrystallization causes smaller $a$. This supports the results in Figures 2 and 3.

For 70/30 SPS/PPO blend, the $T_{\mathrm{c}}$ dependence of $a$ is opposite to that for pure SPS ; $a$ at $T_{\mathrm{c}}=220^{\circ} \mathrm{C}$ is smaller than that at $T_{\mathrm{c}}=240^{\circ} \mathrm{C}$. This suggests that, at $T_{\mathrm{c}}=220^{\circ} \mathrm{C}$, a smaller amount of PPO is entrapped between SPS crystalline and recrystallization is induced between the primary lamellae. However, at $T_{\mathrm{c}}=240^{\circ} \mathrm{C}$, the higher 
concentration of PPO between primary lamellae is entrapped between SPS crystalline so that recrystallization is expected to be strongly suppressed. This may be supplementary support for Figures 2 and 3.

\section{Structure on the Spherulitic Level}

To understand SPS recrytallization and impurity location, the texture of the spherulite was investigated by polarizing optical microscopy. In pure SPS, the compact and small spherulites are formed at $T_{\mathrm{c}}=220^{\circ} \mathrm{C}$ (Figure 7a), whereas the coarse and large spherulites are formed at $T_{\mathrm{c}}=240^{\circ} \mathrm{C}$ (Figure $7 \mathrm{a}^{\prime}$ ). By blending PPO (Figures $7 \mathrm{~b}$ and $\left.7 b^{\prime}\right)$, the tendency of the spherulite texture is reversible relative to that in pure SPS. The texture of the spherulite may be characterized by its compactness, as follows.

In miscible blends of crystalline polymer, the noncrystalline component is rejected from crystallites. The degree of rejection may depend upon the relative rates $(D / G)$ of the diffusion of non-crystalline component $(D)$ and growth of the crystalline component $(G)$.

1. If the value of $D / G$ is comparable with the interlamellar distance, then the rejected non-crystalline impurity may reside between lamellae.

2. If $D$ is larger or $G$ smaller, then $D / G$ may be sufficiently large so that the rejected impurity resides between crystalline fibrils consisting of lamellae.

To investigate whether mode 1 or 2 occurs, the texture of a spherulite may be characterized by its compactness. The coarse structure is characterized by interfibril rejection of non-crystallizable component, whereas the compact structure occurs when the rejection is between individual lamellae stack. ${ }^{13-17}$ Thus, the compact spherulitic appearance in pure SPS crystallized at $T_{\mathrm{c}}=220^{\circ} \mathrm{C}$ suggests that mode 1 occurs and the rejected impurity resides between lamellae. The rejected impurity may retard recrystallization between primary lamellae, supporting the results in previous section. The coarse spherulitic appearance at $240^{\circ} \mathrm{C}$ suggests that the mode 2 occurs and the rejected impurity (i.e., noncrystallizable low molecular weight in pure SPS) resides between fibrils consisting of lamellar bundle.

By blending PPO, the tendency of the spherulitic texture is reversible relative to that in pure SPS. The coarse spherulite at $T_{\mathrm{c}}=220^{\circ} \mathrm{C}$ suggests that the mode 2 occurs and the rejected PPO resides between fibrils consisting of lamellar bundle. The residence of the PPO between interfibrils may induce the recrystallization between the primary lamellae. The compact spherulite is seen at $T_{\mathrm{c}}=240^{\circ} \mathrm{C}$ suggesting that mode 1 occurs and the rejected impurity resides between lamellae. The rejected impurity may retard the recrystallization between primary lamellae. The above results by optical microscopy may be additional evidence for the recrystallization in Figures 2 and 3.

\section{CONCLUSION}

The thermal behavior of the pure SPS and 70/30 SPS/ PPO blend after isothermal crystallization was examined by DSC. Either sharp multiple endotherms or single melting endotherm was observed for SPS and the blend, and the appearance of the traces was dependent on $T_{\mathrm{c}}$. By a series of crystalline morphology by SAXS and polarizing optical microscopy, it was revealed that the multiple or single melting endotherm is deeply related to the position of the rejected impurity (PPO or noncrystallizable chains with low molecular weight). The impurity rejection between primary crystalline lamellae retarded recrystallization and induced the single endotherm, whereas the impurity between crystalline fibrils caused the recrystallization between the primary lamellae giving rise to multiple endotherms during DSC.

\section{REFERENCES}

1. N. Ishihara, T. Seimiya, M. Kuramoto, and M. Uoi, Macromolecules, 19, 2464 (1986).

2. G. Guerra, V. M. Vitagliano, C. DeRosa, V. Petraccone, and P. Corradini, Macromolecules, 23, 1539 (1990).

3. Y. K. Wang, J. D. Savage, D. Yang, and S. L. Hsu, Macromolecules, 25, 3659 (1992).

4. Y. Chatani, Y. Shimane, T. Inagaki, T. Ijitsu, T. Yukinari, and H. Shikuma, Polymer, 34, 1620 (1993).

5. G. Guerra, C. Derosa, V. M. Vitagliano, V. Petraccone, P. Corradini, and F. E. Karasz, Polym. Comm., 29, 265 (1991).

6. J. J. Koberstein, B. Morra, and R. S. Stein, J. Appl. Crystallogr., 13, 34 (1980).

7. F. J. Hybart and J. D. Platt, J. Appl. Polym. Sci., 49, 247 (1991).

8. B. S. Hsiao, K. H. Gardner, D. Q. Wu, and B. Chu, Polymer, 34, 3996 (1993).

9. R. C. Roberts, Polymer, 10, 117 (1969).

10. G. R. Strobl, M. J. Schneider, and I. G. Voigt-Martin, J. Polym. Sci., Polym. Phys. Ed., 18, 1361 (1980).

11. H. L. Chen, J. C. Hwang, and C. C. Chen, Polymer, 37, 5461 (1996).

12. P. Debye, H. R. Anderson, and H. Brumberger, J. Appl. Phys. 28, 6 (1957).

13. H. D. Keith and F. J. Padden, Jr., J. Appl. Phys., 35, 1270 (1964).

14. H. D. Keith and F. J. Padden, Jr., J. Appl. Phys., 34, 2409 (1963).

15. F. P. Warner, W. J. Macknight, and R. S. Stein, J. Polym. Sci., Polym. Phys. Ed., 15, 2113 (1997).

16. R. S. Stein, F. B. Khambatta, F. P. Warner, T. P. Russell, A. Escala, and E. Balizer, J. Polym. Sci., Polym. Phys. Ed., 63, 313 (1978).

17. S. D. Hudson, D. D. Davis, and A. J. Lovinger, Macromolecules, 25, 1759 (1992). 